\title{
Fluoride content of UK retail tea: impact of brew time on teas of different value
}

\author{
C.H.S. Ruxton ${ }^{1}$ and T.J. Bond ${ }^{2}$ \\ ${ }^{1}$ Freelance Dietitian, Nutrition Communications, 26 East Road, Cupar, KY15 4HQ and ${ }^{2}$ Technical expert, \\ Tea Advisory Panel, 52a Cromwell Road, London SW7 5BE
}

Tea is a natural source of fluoride (F-) and a major contributor to UK F- intakes. F- has well established oral health benefits ${ }^{(1)}$. We previously ${ }^{(2)}$ reported the F- content of 49 retail teas, finding wide variation in results and a lower F- in speciality and single estate teas. Other work suggested that economy tea bags are higher in $\mathrm{F}_{-}{ }^{(3)}$. As our original study, and an intervention confirming the hydrating benefits of tea ${ }^{(4)}$, used a 40 s brewing time, a pilot study was done to assess differences in the F- content of black tea brewed for longer periods.

Three brands of retail black tea were selected to represent economy, mid-range and premium products. Infused F- was measured by brewing one tea bag in $240 \mathrm{ml}$ of freshly boiled de-ionized water for the required period, before squeezing the tea bag gently against the side of the beaker and removing it. This was repeated in triplicate and the individual brews combined. The combined brews were then stirred and cooled to approximately $20^{\circ} \mathrm{C}$ before aliquots were taken and the F-content measured using ion selective electrode. The entire process was carried out in triplicate for each brand and each brewing time of 40, 120, 240 and 360 seconds. The results are shown below per $240 \mathrm{ml}$ serving. Between-subjects and repeated ANOVA were used to test for significant differences $(\mathrm{p}<0 \cdot 05)$.

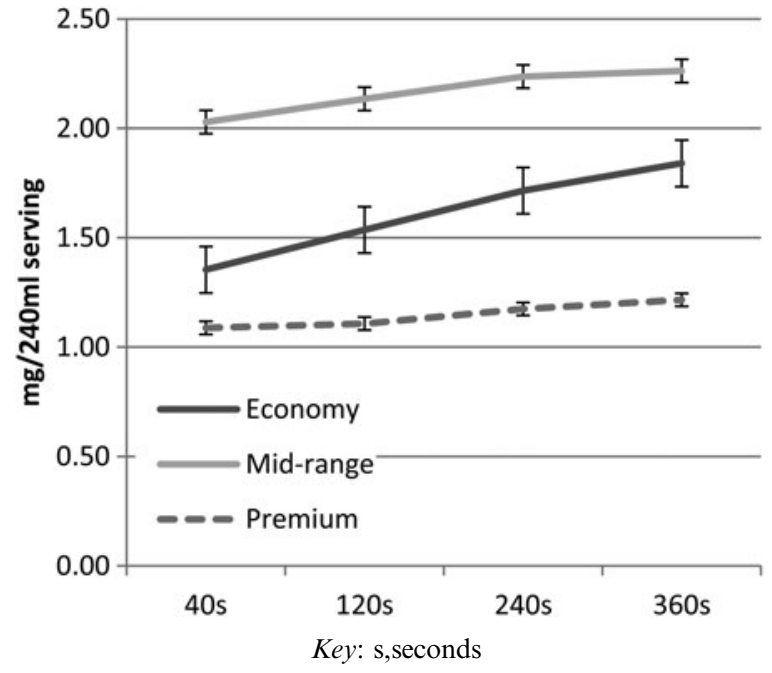

F- content was significantly different at 40 s with the highest value seen in the mid-range tea. F- content significantly increased with brewing time for all three individual brands. However, the increase in F-content between 40s and 360s was greatest in the economy tea $(+2.03 \mathrm{mg} / \mathrm{L})$ compared with the premium tea $(+0.53 \mathrm{mg} / \mathrm{L})$. In conclusion, brewing time has a significant impact on the F- content of tea, particularly for economy tea. This may be because economy teas contain a higher proportion of lower graded tea leaves which have a smaller particle size. The effect of this would be a more rapid infusion. In contrast, premium teas contain younger leaves which have had less time to accumulate F- from the soil.

This work was funded by the Tea Advisory Panel, which is supported by an unrestricted educational grant from the UK Tea \& Infusions Association.

1. European Commission (2012) Official Journal of the European Union L136, 1-40.

2. Ruxton CHS \& Bond TJ (2014) Proc Nutr Soc In press.

3. Chan L, Mehra A, Saikat S et al. (2013) Food Res Int 51, 564-70.

4. Ruxton CHS \& Hart VA (2011) Br J Nutr 106, 588-95. 